Journal of Mathematics and Statistics 3 (2): 65-69, 2007

ISSN 1549-3644

(C) 2007 Science Publications

\title{
Induction Motor Flux Estimation using Nonlinear Sliding Observers
}

\author{
${ }^{1}$ Hakiki Khalid, ${ }^{2}$ Mazari Benyounès and ${ }^{1}$ Djaber Sid'Ahmed \\ ${ }^{1}$ Laboratoire d'Automatique et d'Analyse des Systèmes (L.A.AS)ENSET-Oran \\ BP 1523 El Mnaouer Oran Algerie \\ ${ }^{2}$ Laboratoire de Développement des Entraînements Electriques (LDEE)USTOMB-Oran
}

\begin{abstract}
A nonlinear sliding flux was proposed for an induction motor. Its dynamic observation errors converge asymptotically to zero, independently from the inputs. The aim of this work was to study the robustness of this observer with respect to the variation of the rotor resistance known to be a crucial parameter for the control. The dynamic performance of this sliding observer was compared to that of Verghese observer via a simulation of an IM driven by U/F control in open loop.
\end{abstract}

Key words: Nonlinear sliding observer, induction motor, rotor flux estimation

\section{INTRODUCTION}

DC motors have been used extensively in the industry because of the simple control strategies required to achieve good performance, in variable speed applications. However, in comparison with their counterparts, IM drives, DC drives result more expensive and less robust devices, not to mention the maintenance they require due to the commutator. Because they are highly nonlinear, thus requiring much more control complex algorithms, IM drives were rarely used in control applications in the past.

Nowadays, as a consequence of the important progress realized in nonlinear control theory and power electronics, the AC drives, by using new control techniques, have proved to outperform the DC ones. Among these techniques, both field oriented control (FOC) and nonlinear input-output decoupling have emerged as powerful tools for high performance control of induction machines ${ }^{[1,2]}$. The main drawback of both algorithms is the need of flux sensors, which are to be inserted in the air gap and involve a redesign of the machine, which reduces reliability and implies both additional costs and technological difficulties. For this reason, flux observers have been widely investigated ${ }^{[3,4]}$ : they are rather sensitive with respect to rotor resistance variations.

Starting with ${ }^{[5]}$, rotor resistance estimators have been studied ${ }^{[5,6]}$ but most contributions rely on simplifying assumptions and definite results are still not completely available since, no method applies when the motor is in low-speed regime.
In the literature, several alternative methods exist for the design of different observer structures: linearization by a change of coordinates and output injection $^{[7-9]}$, variable structure systems ${ }^{[10]}$ and Lyapunov-based design ${ }^{[11]}$. However, closed-loop stability cannot be guaranteed a priori if the control design is based on the separation principle, which is verified only for linear systems. Thus, based on control theory and noting that observability is a dual problem of controllability, sliding mode observers were developed ${ }^{[12-14]}$. They derive from a transposition of the switching controllers ${ }^{[12]}$ to the problem of state observation in nonlinear systems. Sliding control design consists in defining a switching surface in the phase plane that is rendered attractive by the action of the switching terms. The dynamics is determined by the Fillipov solution concept ${ }^{[15]}$, which indicates that the system dynamic behaviour within the switching surface can be described as a pondered average of the dynamics of each side of the discontinuous surface.

Class of sliding mode observers: Consider the $n^{\text {th }}$ order nonlinear system:

$\dot{x}=f(x, u), \quad x \in R^{n} ; \quad u=R^{q}$

and for convenience, consider a vector of measurements:

$y=C x, \quad y \in R^{r}$

The system is assumed to be observable and the observer is defined with the following structure :

$\dot{\hat{x}}=\hat{f}(\hat{x}, y, u)+K I_{s}$ 
where $\hat{x} \in R^{n}, \hat{f}$ is our model of $f, \mathrm{~K}$ is $n \times r$ gain matrix to be specified and

$$
I_{s}=\left[\operatorname{sgn}\left(s_{1}\right), \operatorname{sgn}\left(s_{2}\right), \ldots . ., \operatorname{sgn}\left(s_{r}\right)\right]^{T}
$$

where

$$
\left[s_{1}, s_{2}, \ldots . ., s_{r}\right]^{T}=S=\Gamma[y-C \hat{x}]
$$

and $\Gamma$ is $r \times q$ matrix to be specified. Defining the error vector $\tilde{y}=C \tilde{x}$ and $\tilde{x}=(x-\hat{x})$, one has

$$
\dot{\tilde{x}}=\Delta f-K I_{s}
$$

where $\Delta f=f(x, u)-\hat{f}(\hat{x}, y, u)$

The $r$ dimensional surface $\mathrm{S}=0$ will be attractive

if: $s_{i} \dot{s}_{i}<0, i \in\{1, \ldots . r\}$ During the sliding, the switching term in (4) is keeping $S \equiv 0$; hence, formally $S \equiv 0$. So, $\tilde{I}_{s}$, the equivalent switching vector ${ }^{[16]}$ can be obtained from: $\Gamma \mathrm{C}\left(\Delta \mathrm{f}-\mathrm{K} \tilde{\mathrm{I}}_{\mathrm{S}}\right)=0$

so that: $\tilde{I}_{s}=(\Gamma C K)^{-1} \Gamma C \Delta f$

The $r \times r$ matrix $\Gamma C K$ is invisible with an appropriate choice for $\Gamma$ and K. Thus, from (6) and (7) the equivalent dynamics on the reduced order manifold is given by:

$$
\dot{\tilde{x}}=\left(I-K(\Gamma C K)^{-1} \Gamma C\right) \Delta f
$$

with $\Gamma C \tilde{x}=0$ The structure of $\Delta f$ must be known before any further analysis can be done

Verghese observer: The dynamic behaviour of an induction motor working under no saturation of its magnetic circuits can be described in a fixed stator reference $(\alpha-\beta)$ frame ${ }^{[2]}$ by:

$$
\left\{\begin{array}{l}
d \phi_{\alpha, r} / d t=-b \phi_{\alpha, r}+a i_{\alpha, s}-\omega p \phi_{\beta, r} \\
d \phi_{\beta, r} / d t=-b \phi_{\beta, r}+a i_{\beta, s}+\omega p \phi_{\alpha, r} \\
d i_{\alpha, s} / d t=\gamma_{4} V_{\alpha, s}-\gamma_{1} i_{\alpha, s}+\gamma_{2} \phi_{\alpha, r}+\gamma_{3} \omega \phi_{\beta, r} \\
d i_{\beta, s} / d t=\gamma_{4} V_{\beta, s}-\gamma_{1} i_{\beta, s}+\gamma_{2} \phi_{\beta, r}-\gamma_{3} \omega \phi_{\beta, r} \\
J_{r} d \omega / d t=p\left(M / L_{r}\right)\left[i_{\beta, s} \phi_{\alpha, r}-i_{\alpha, s} \phi_{\beta, r}\right]-T_{L}+f_{v} \omega
\end{array}\right.
$$

with $\alpha_{1}=p M / J_{r} L_{r}$, $b=R_{r} / L_{r}=1 / T_{r} \sigma=1-\left(M^{2} / L_{s} L_{r}\right), a=R_{r} M / L_{r}$ $\gamma_{1}=\left(R_{s} / \sigma L_{s}\right)+\left(R_{r} M^{2} / \sigma L_{s} L_{r}^{2}\right) \gamma_{2}=M R_{r} / \sigma L_{s} L_{r}^{2}$, $\gamma_{3}=M p / \sigma L_{s} L_{r}, \gamma_{4}=1 / \sigma L_{s}$

where: $\quad\left(\phi_{\alpha, r}, \phi_{\beta, r}\right),\left(i_{\alpha, s}, i_{\beta, s}\right), T_{L}, \quad J_{r}, \sigma, \quad f_{v}$, $R_{r}, L_{r}, R_{s}, L_{s}, M, T_{r}, \omega$ and $p$ are respectively the rotor fluxes, the stator currents, the torque load, the moment of inertia, the leakage and sticky friction coefficients, the rotor and stator winding resistances and inductances, the mutual inductance, the rotor time constant, the mechanical speed and the number of pole pairs. Therefore, setting $x=\left(\omega, \phi_{\alpha, r}, \phi_{\beta, r}, i_{\alpha, s}, i_{\beta, s}\right)^{T}$, (9) is written in the form:

$$
\left\{\begin{array}{l}
\dot{x}_{1}=\alpha_{1}\left(x_{5} x_{2}-x_{3} x_{4}\right)-\alpha_{2} T_{L}-\alpha_{3} x_{1} \\
\dot{x}_{2}=a x_{4}-b x_{2}-p x_{1} x_{3} \\
\dot{x}_{3}=a x_{5}-b x_{3}+p x_{1} x_{2} \\
\dot{x}_{4}=-\gamma_{1} x_{4}+\gamma_{2} x_{2}+\gamma_{3} x_{1} x_{3}+\gamma_{4} v_{1} \\
\dot{x}_{5}=-\gamma_{1} x_{5}+\gamma_{2} x_{3}-\gamma_{3} x_{1} x_{2}+\gamma_{4} v_{2}
\end{array}\right.
$$

The Verghese observer model which is a copy of the first four equations of (9) where added a corrective term due to a prediction error, is written in compact form $\mathrm{as}^{[3]}$ :

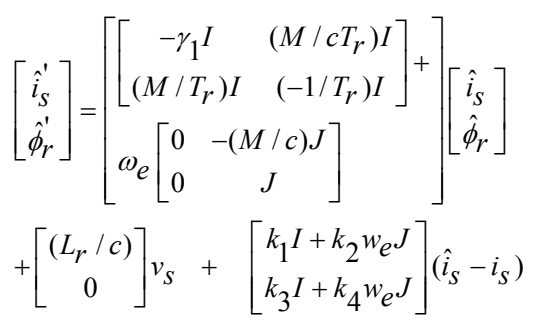

where

$\phi_{r}=\left[\phi_{\alpha, r} \phi_{\beta, r}\right]^{T}, i_{s}=\left[\begin{array}{ll}i_{\alpha, s} & i_{\beta, s}\end{array}\right]^{T}, v_{s}=\left[V_{\alpha, s}, V_{\beta, s}\right]^{T}$,

$I=\left[\begin{array}{ll}1 & 0 \\ 0 & 1\end{array}\right], \quad J=\left[\begin{array}{cc}0 & -1 \\ 1 & 0\end{array}\right], \quad c=\sigma L_{s} L_{r}$

$\left(\hat{i}_{s}^{\prime}, \hat{\phi}_{r}^{\prime}\right)$ are the derivatives of $\left(\hat{i}_{s}, \hat{\phi}_{r}\right)$ estimates of $\left(i_{s}, \phi_{r}\right), k_{i}$ are scalars and $\omega_{e}=p \omega$ is the electrical speed of the rotor. The dynamics of the observation error $e=\hat{x}-x$ is given by :

$$
e^{\prime}=\left[\begin{array}{c}
{\left[\begin{array}{cc}
\left(k_{1}-\gamma_{1}\right) I & \left(M / c T_{r}\right) I \\
{\left[k_{3}+\left(M / T_{r}\right)\right] I} & \left(-1 / T_{r}\right) I
\end{array}\right]} \\
+\omega_{e}\left[\begin{array}{cc}
k_{2} J & -(M / c) J \\
k_{4} J & J
\end{array}\right]
\end{array}\right] e .
$$

If $k_{1}$ and $k_{3}$ are selected such that: $k_{1}-\gamma_{1}=-k_{2} / T_{r}$ and $k_{3}+\left(M / T_{r}\right)=-k_{4} / T_{r}$

the error dynamics becomes : $e^{\prime}=A Q e$

where: $A=\left[\begin{array}{cc}k_{2} I & -(M / c) I \\ k_{4} I & I\end{array}\right]$

and: $Q=\left[\begin{array}{cc}\left(-1 / T_{r}\right) I+\omega_{e} J & 0 \\ 0 & \left(-1 / T_{r}\right) I+\omega_{e} J\end{array}\right]$.

The freedom that one has in choosing $k_{2}$ and $k_{4}$ is used to place the eigenvalues of A in pairs at arbitrary locations, as is verified by noting that the characteristic polynomial of $\mathrm{A}$ is:

$$
\left[p^{2}-\left(1+k_{2}\right) p+k_{2}+k_{4}(M / c)\right]^{2}
$$


If the eigenvalues of $\mathrm{A}$ are $p_{1}$ (twice) and $\mathrm{p}_{2}$ (twice), then the eigenvalues of the matrix product in (11) can be shown to be:

$\left[\left(-1 / T_{r}\right) \pm j \omega_{e}\right] p_{1}$ and $\left[\left(-1 / T_{r}\right) \pm j \omega_{e}\right] p_{2}$

Hence, if the speed is (nearly) constant, the error dynamics is ( approximately) governed by these eigenvalues. If it is time-varying, we will attempt a Lyapunov analysis.

Flux sliding mode observer: The proposed type of sliding mode based observer of (9) can be written as:

$$
\left\{\begin{array}{l}
\dot{\hat{x}}_{1}=\alpha_{1}\left(x_{5} \hat{x}_{2}-\hat{x}_{3} x_{4}\right)-\alpha_{2} T_{L}-\alpha_{3} x_{1}+K_{1} I_{s}+q_{1}\left(x_{1}-\hat{x}_{1}\right) \\
\dot{\hat{x}}_{2}=a x_{4}-b \hat{x}_{2}-p x_{1} \hat{x}_{3}+K_{2} I_{s} \\
\dot{\hat{x}}_{3}=a x_{5}-b \hat{x}_{3}+p x_{1} \hat{x}_{2}+K_{3} I_{s} \\
\dot{\hat{x}}_{4}=-\gamma_{1} x_{4}+\gamma_{2} \hat{x}_{2}+\gamma_{3} x_{1} \hat{x}_{3}+\gamma_{4} v_{1}+K_{4} I_{s} \\
\dot{\hat{x}}_{5}=-\gamma_{1} x_{5}+\gamma_{2} \hat{x}_{3}-\gamma_{3} x_{1} \hat{x}_{2}+\gamma_{4} v_{2}+K_{5} I_{s}
\end{array}\right.
$$

where $K_{i} I_{s}=\lambda_{i 1} \operatorname{sign}\left(s_{1}\right)+\lambda_{i 2} \operatorname{sign}\left(s_{2}\right)$. for $i \in\{1, \ldots, 5\}$, $K_{i}$ and $q_{1}$ are the observers gains. The sliding surface $\mathrm{S}$ is given by:

$$
S=M\left[\begin{array}{l}
x_{4}-\hat{x}_{4} \\
x_{5}-\hat{x}_{5}
\end{array}\right]=\left[\begin{array}{l}
s_{1} \\
s_{2}
\end{array}\right]=\left[\begin{array}{l}
0 \\
0
\end{array}\right]
$$

Setting $e_{i}=x_{i}-\hat{x}_{i}$ for $i \in\{1, \ldots ., 5\}$, the observation error dynamics is:

$$
\left\{\begin{array}{l}
\dot{e}_{1}=\alpha_{1}\left(x_{5} e_{2}-e_{3} x_{4}\right)-K_{1} I_{s}-q_{1} e_{1} \\
\dot{e}_{2}=-b e_{2}-p x_{1} e_{3}-K_{2} I_{s} \\
\dot{e}_{3}=-b e_{3}+p x_{1} e_{2}-K_{3} I_{s} \\
\dot{e}_{4}=\gamma_{2} e_{2}+\gamma_{3} x_{1} e_{3}-K_{4} I \\
\dot{e}_{5}=\gamma_{2} e_{3}-\gamma_{3} x_{1} e_{2}-K_{5} I_{s}
\end{array}\right.
$$

The stability analysis consists of determining $K_{4}$ and $K_{5}$ such that the surface $S \equiv 0$ is the attractive. Then $K_{1}, \mathrm{~K}_{2}, K_{3}$ and $q_{1}$ are determined such that the reduced order system obtained when $S \equiv 0$ is locally stable to 0 in the attractive domain defined as follows: Let us consider the Lyapunov function $\mathrm{V}=\frac{\mathrm{S}^{\mathrm{T}} \mathrm{S}}{2}$ such that $\left(\mathrm{S}=0 \rightarrow \mathrm{e}_{4}=\mathrm{e}_{5}=0\right)$ with $\mathrm{M}$ as a regular matrix. The attractive of sliding surface $\mathrm{S}=0$ is given by:

$$
\forall \mathrm{S} \neq 0, \dot{\mathrm{V}}=\mathrm{S}^{\mathrm{T}} \frac{\partial \mathrm{S}}{\partial \mathrm{t}}=\mathrm{S}^{\mathrm{T}} \mathrm{MW}<0
$$

where: $\quad \mathrm{W}=\mathrm{M}^{-1}\left[\begin{array}{l}\mathrm{e}_{2} \\ \mathrm{e}_{3}\end{array}\right]-\left(\begin{array}{ll}\lambda_{41} & \lambda_{42} \\ \lambda_{51} & \lambda_{52}\end{array}\right) \mathrm{I}_{\mathrm{S}} \quad$ with: $\left[\begin{array}{l}\mathrm{K}_{4} \\ \mathrm{~K}_{5}\end{array}\right]=\mathrm{M}^{-1} \Delta$; $\Delta=\left[\begin{array}{cc}\delta_{1} & 0 \\ 0 & \delta_{2}\end{array}\right], \delta_{1}, \delta_{2}>0$.
From the singular perturbation theory ${ }^{[14]}$, the dynamics of $\omega$ is supposed to be a slow variable with respect to the currents and the flux dynamics. The conditions of attraction between $\mathrm{s}_{1}, \mathrm{e}_{2}$ and $\mathrm{s}_{2}, \mathrm{e}_{3}$ are decoupled . So (12) is obtained within the set defined by the following inequalities:

$\left\{\begin{array}{l}\text { if } \mathrm{s}_{1}>0 \text { then } \mathrm{e}_{2}<\delta_{1} \\ \text { if } \mathrm{s}_{1}<0 \text { then } \mathrm{e}_{2}>-\delta_{1} \\ \text { if } \mathrm{s}_{2}>0 \text { then } \mathrm{e}_{3}<\delta_{2} \\ \text { if } \mathrm{s}_{2}<0 \text { then } \mathrm{e}_{3}>-\delta_{2}\end{array}\right.$

On the sliding surface, $\mathrm{S}=0$ which is invariant, the vector $\stackrel{\Delta}{\mathrm{I}_{\mathrm{S}}}=\tilde{\mathrm{I}}_{\mathrm{S}}$ is given by:

$\left(\begin{array}{l}0 \\ 0\end{array}\right)=M^{-1}\left[\left(\begin{array}{l}e_{2} \\ e_{3}\end{array}\right)-\left(\begin{array}{cc}\delta_{1} & 0 \\ 0 & \delta_{2}\end{array}\right) I_{S}\right]$ and $\tilde{I}_{\mathrm{S}}=\left(\begin{array}{l}\mathrm{e}_{2} / \delta_{1} \\ \mathrm{e}_{3} / \delta_{2}\end{array}\right)=\Delta^{-1} \mathrm{e}$

From the definition of equivalent vector ${ }^{[16]}$, one obtains the dynamics error after a finite time $t_{0}$ which is reduced to: $e_{1}=\alpha_{1}\left(x_{5} e_{2}-e_{3} x_{4}\right)-\left(\begin{array}{ll}\lambda_{11} / \delta_{1} & \lambda_{12} / \delta_{2}\end{array}\right)\left(\begin{array}{l}e_{2} \\ e_{3}\end{array}\right)_{s}-q_{1} e_{1}$ $\left(\begin{array}{l}\dot{\mathrm{e}}_{2} \\ \dot{\mathrm{e}}_{3}\end{array}\right)=\left[\mathrm{H}-\left(\begin{array}{cc}\lambda_{21} & \lambda_{22} \\ \lambda_{31} & \lambda_{32}\end{array}\right) \Delta^{-1}\right]\left(\begin{array}{l}\mathrm{e}_{2} \\ \mathrm{e}_{3}\end{array}\right), \mathrm{H}=\left(\begin{array}{cc}-\mathrm{b} & -\mathrm{p} \mathrm{x}_{1} \\ \mathrm{px}_{1} & -\mathrm{b}\end{array}\right)$

Thus: $\left[\begin{array}{ll}\lambda_{11} / \delta_{1} & \lambda_{12} / \delta_{2}\end{array}\right]=\left[\begin{array}{ll}\alpha_{1} \mathrm{x}_{5} & \left.\alpha_{1} \mathrm{x}_{4}\right)\end{array}\right]$ and :

$\left(\begin{array}{ll}\lambda_{21} & \lambda_{22} \\ \lambda_{31} & \lambda_{32}\end{array}\right)=\left[\mathrm{H}+\left(\begin{array}{cc}\mathrm{q}_{2} & 0 \\ 0 & \mathrm{q}_{3}\end{array}\right)\right] \Delta$

The reduced system of the observer errors can be written as: $\dot{e}_{i}=-q_{i} e_{i}$ with $q_{i}>0$ and $i=1,2,3$ The observation dynamics error is then stable, with an appropriate choice of $q_{i}$

Digital simulation: A combination of the two observers presented in Sections 3 and 4, is simulated in Matlab/Simulink for the simultaneous estimation of rotor fluxes. The combined estimation is depicted in the block diagram of Fig. 1 


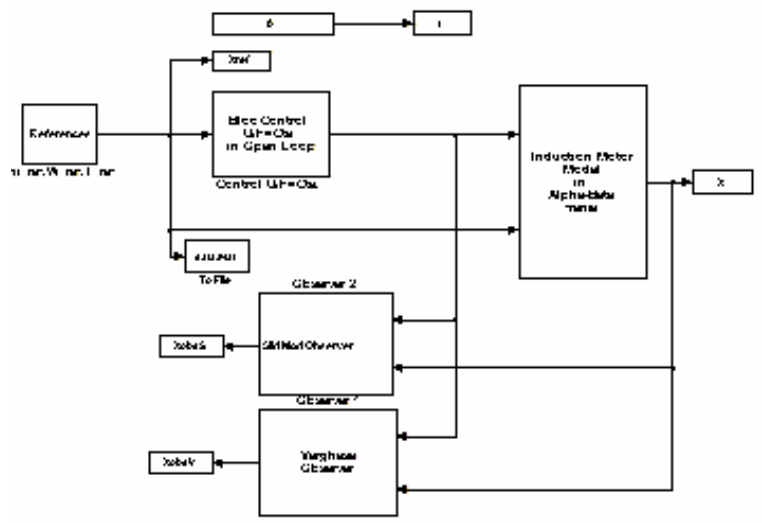

Fig. 1: Flux observers for induction motor
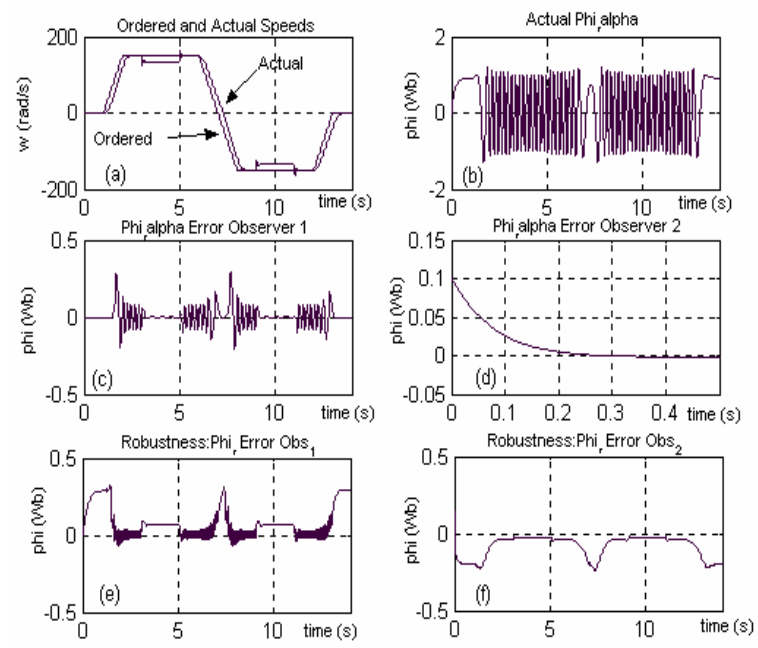

Fig. 2: Simulation results

A digital simulation of the proposed combined scheme illustrates its behaviour when the motor is operating under U/F control in open loop with full load capability (The IM data in simulation are given in the Appendix).

Figure 2a shows the reference speed of the motor with the measured one and Fig. $2 b$ shows the first component of the rotor flux .

From Fig. 2c, we verify that observation errors converge to zero in steady-state operations but, as stated previously, they tend to their maximum value, in low-speed regime, particularly at zero-crossing. Figure $2 \mathrm{~d}$ illustrates the well-known insensitivity property of sliding modes with respect to disturbances.

Figure $2 \mathrm{e}$ and $2 \mathrm{f}$ show the robustness of the two observers in case of an instantaneous rotor resistance increase of $50 \%$ in the IM drive.

\section{CONCLUSION}

In this study, a novel combined scheme for concurrent estimation of the rotor fluxes of an IM was presented. The proposed method is based on two nonlinear observers. The simulation results show a good performance of the sliding mode estimation scheme.

It has been shown that sliding mode observer design methods based on the prescribed form of the Lyapunov function candidate can be successfully applied. The simulation results are suggesting that design can be implemented based on the mechanical motion measurement only, thus avoiding flux variable measurement. In addition, the simplicity of the algorithm makes it suitable for an on-line implementation.

In further work, the authors intend to study highorder sliding modes, in both control and observation for an induction machine and a pneumatic robot, to remove the chattering effect which is known to be the main drawback of the standard sliding modes.

\section{ACKNOWLEDGEMENTS}

The present research work has been supported by Science and Technology for Safety in Transportation and funded by the European Union, the Délégation Régionale à la Recherche et à la Technologie, the Ministère Délégué à l'Enseignement Supérieur et à la Recherche, the Région Nord Pas de Calais (France) and the Centre National de la Recherche Scientifique.

\section{Appendix}

Total Stator Inductance Machines parameters

Total Rotor Inductance $\quad 0.142 \mathrm{H}$

Mutual Inductance $\quad 0.099 \mathrm{H}$

Stator Resistance $\quad 1.633 \Omega$

Rotor Resistance $\quad 0.93 \Omega$

Rotor Inertia (IM + load) $\quad .029 \mathrm{Kgm}^{2}$

Number of Pole pairs

Rated magnitudes

Direct voltage

2

Load Torque $\quad+7 \&-7 \mathrm{Nm}$

Speed $1430 \mathrm{rpm}$

Stator Flux $\quad 0.59 \mathrm{~Wb}$

Power $\quad 1.5 \mathrm{Kw}$

Coefficient of sticky friction $\quad 0.0038 \mathrm{Nms} / \mathrm{rd}$ 


\section{REFERENCES}

1. Vas, P., 1994. Vector control of AC Machines. Oxford Science Publications, New York, USA.

2. Marino, R. S. Peresada and P. Valigi, 1993. Adaptive input-output linearizing control of induction motors. IEEE Trans. Automatic. Control, 38: 208-221.

3. Verghese, G.C. and S.R. Sanders, 1988. Observers for flux estimation in induction machines. IEEE Trans. Ind. Electron, 35: 85-94.

4. Du, T. and M. Brdys, 1993. Implementation of extended Luenberger observers for joint state and parameter estimation of PWM induction motor drives. Proc. EPE, Brighton UK.

5. Garces, L.J., 1980. Parameter adaptation for the speed controlled static ac drive with a squirrel cage induction motor. IEEE Trans Ind. Applicat., IA-16: 173-178.

6. Friedland, B., 1997. A nonlinear observer for estimating parameters in dynamic systems. Automatica, 33: 1525-1530.

7. Krener, A. and A. Isidori, 1983. Linearization by output injection and nonlinear observers. Systems and Control Lett., 3: 47-52.

8. Boukhobza, T., M. Djemai and J.P. Barbot, 1996. Nonlinear sliding observer for systems in output and output derivatives injection form. IFAC World Congress, San Francisco, CA.
9. Hakiki, K., B. Mazari and S. Djaber, 2003. Observateur par modes glissants sous forme triangulaire à injection de sortie d'un robot pneumatique. $1^{\text {er }}$ Congres International sur la modélisation numérique appliquée CIMNA 1, 1415 Nov.

10. Edwards, C. and C.P. Tan, 2006. Sensor fault tolerant control using sliding mode observers. J. Control Engg. Practice, 14: 897-908.

11. Thau, F.E., 1973. Observing the state of nonlinear dynamic systems. Int. J. of Contr., 17: 441-479.

12. Utkin, V.I., 1992. Sliding Modes in Control Optimisation. New York: Springer-Verlag.

13. Drakunov, S. and V. Utkin, 1995. Sliding mode observer tutorial. Proc. $34^{\text {th }}$ IEEE Conf. on Decision and Control, pp: 3376-3379.

14. Floquet, T. and J.P. Barbot, 2004. A sliding mode approach of unknown input observers for linear systems. $43^{\text {rd }}$ IEEE Conf. Decision and Control, Dec. 14-17, pp: 1724-1729.

15. Fillipov, A.F., 1960. Differential equations with discontinuous right-hand side. Am. Math. Soc. Trans., 62: 199-231.

16. Kokotovic, P., H.K. Khalil and J.O' Reilly, 1999. Singular Perturbation methods in Control, Analysis and Design. Society for Industrial and Applied Mathematics, Academic Press. 